\title{
ARTICLE PPAR $\gamma$ enhances ILC2 function during allergic airway inflammation via transcription regulation of ST2
}

\author{
Qiang Xiao ${ }^{1,2}$, Juan $\mathrm{He}^{1,3}$, Aihua Lei ${ }^{4}$, Haixu Xu ${ }^{1}$, Lijuan Zhang ${ }^{1}$, Pan Zhou ${ }^{1}$, Guanmin Jiang ${ }^{2}$ and Jie Zhou (D)
}

\begin{abstract}
Group 2 innate lymphoid cells (ILC2s) represent the major player during hyperresponsive airway inflammation. Peroxisome proliferator-activated receptor- $\gamma$ (PPAR $\gamma$ ) was highly expressed on ILC2 and its potential role in asthma has been suggested. However, the detailed mechanism underlying the effects of PPAR $\gamma$ on ILC2-induced airway inflammation remains to be fully understood. Here we identified PPARY as a positive regulator of lung ILC2. Expression of PPARY on ILC2 was dramatically induced upon interleukin-33 (IL-33) challenge. Deficiency of PPAR in hematopoietic system in mice (PPAR $\gamma^{\mathrm{fl} / \mathrm{fl}}$ Vav1 ${ }^{\mathrm{Cre}}$ ) significantly impaired the function of ILC2 in lung, which led to apparent alleviation of airway inflammation in response to IL-33 or Papain challenge, when compared with those in PPAR $\gamma^{\mathrm{f} / \mathrm{fl}}$ littermates control. Mechanistic studies identified IL-33 receptor ST2 as a transcriptional target of PPARY. Overexpression of ST2 rescued the functional defects of ILC2 lacking PPARy. Collectively, these results demonstrated PPARY as an important regulator of ILC2 during allergic airway inflammation, which sheds new lights on the importance of PPAR $y$ in asthma.
\end{abstract}

Mucosal Immunology (2021) 14:468-478; https://doi.org/10.1038/s41385-020-00339-6

\section{INTRODUCTION}

The incidence of hyperresponsive allergic airway inflammation, or asthma, has increased over recent years. It affects over 300 million of people worldwide. ${ }^{1}$ Over-activation of type 2 helper T cells (Th2) has long been considered to be the dominant mechanism in the pathogenesis of asthma. In the last decade, however, group 2 innate lymphoid cells (ILC2s) were identified as a major player in the initiation of airway inflammation. ${ }^{2}$ Upon allergen exposure, airway epithelium secrete alarmins, including interleukin (IL)-33, IL-25, and thymic stromal lymphopoietin (TSLP), thereby causing the activation of resident ILC2s in lung. ${ }^{2,3}$ Activated ILC2s produce large amounts of type 2 effector cytokines and cause eosinophilic infiltration, goblet cell proliferation, and mucus production, which ultimately lead to airway inflammation. ${ }^{4}$ In addition to the crucial role in the initiation of airway inflammation, the important role of ILC2 $s$ in the facilitation of Th2 activation has also been suggested. 5,6 Therefore, ILC2s could initiate and promote the progression of asthma.

The development of ILC2s happens in the bone marrow: they differentiate from common lymphoid progenitor (CLP), followed by distinct stages of progenitors including $a 4 \beta 7^{+}$lymphoid progenitor ( $a-L P)$, common helper-like ILC progenitor (ChILP), and immature ILC2 ${ }^{7,8}$ After that, ILC2s migrate and reside in the peripheral tissues. ${ }^{9} 10$ Upon stimulations from the tissue microenvironment, such as danger signals, tissue injury or pathogen infections, ILC2s are activated by secreted cytokines, neuropeptides, or hormones. ${ }^{11}$ Activated ILC2s exert a variety of functions in a context or tissue-dependent manner, including but not limited to tissue homeostasis, allergic inflammation, defense against pathogens, as well as energy metabolism. ${ }^{12-14}$ Multiple transcriptional factors and cell-cell interaction molecules have been identified to be important regulators of $\mathrm{ILC} 2,{ }^{8,15}$ among which GATA3 was considered to be the master regulator. ${ }^{16,17}$

Peroxisome proliferator-activated receptor- $\gamma$ (PPAR $\gamma$ ) is a ligandactivated transcription regulator that belongs to the nuclear hormone receptor superfamily. PPARy is ubiquitously expressed in most cell types. Through sensing a wide range of exogenous or endogenous signals from the environment, such as lipid mediators or inflammatory stimuli, PPAR $y$ is activated and regulates the transcription of downstream targets. ${ }^{18,19}$ The importance of PPARY in adipocyte differentiation and lipogenesis has been well-documented. ${ }^{20}$ Its emerging role in the immune system and inflammation is just being recognized. ${ }^{21}$ It was reported that PPARY expression was upregulated during allergen ovalbumin (OVA)-induced airway inflammation. ${ }^{22}$ Furthermore, asthma patients presented higher level of PPARy expression in the airways when compared with healthy controls. ${ }^{23}$ Retrospective studies have also revealed a correlation between PPAR $y$ single nucleotide polymorphisms (SNPs) and the risk of asthma. ${ }^{24,25}$ Clinical trials in both short-term and long-term studies showed a modest effect of PPARy agonists on the alleviation of asthma symptoms in smokers. ${ }^{26}$ Additionally, administration of PPARY agonists exerted strong beneficial effects on murine airway inflammation models. ${ }^{27,28}$ These observations suggested a potential role of PPARY in the pathogenesis of asthma.

The mechanisms underlying the effects of PPARY on airway inflammation remain to be fully elucidated. Both antiinflammatory and pro-inflammatory effects have been reported,

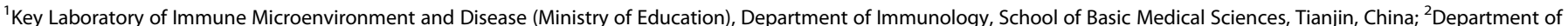

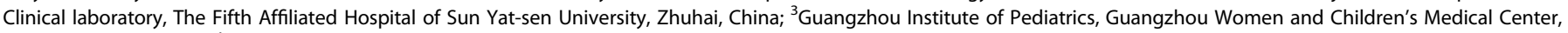
Guangzhou, China and ${ }^{4}$ Institute of Pathogenic Biology, Hengyang Medical College, University of South China, Hengyang, China

Correspondence: Jie Zhou (zhoujie@tmu.edu.cn)

These authors contributed equally: Qiang Xiao, Juan $\mathrm{He}$

Received: 27 May 2020 Revised: 21 July 2020 Accepted: 26 July 2020

Published online: 18 August 2020 
a

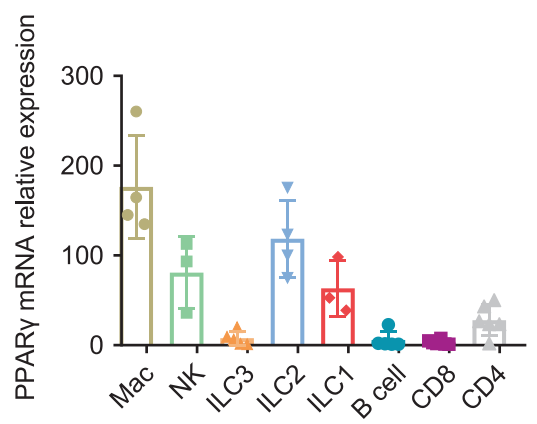

b

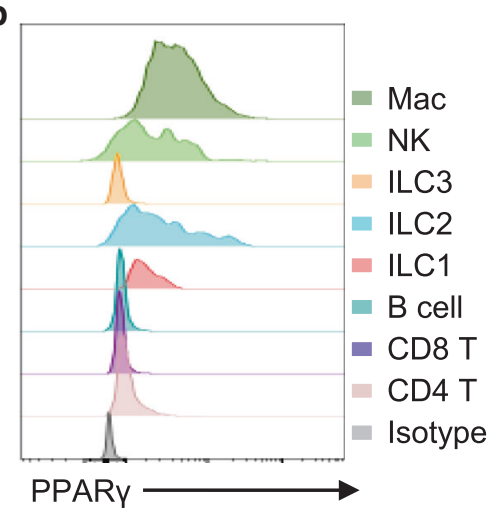

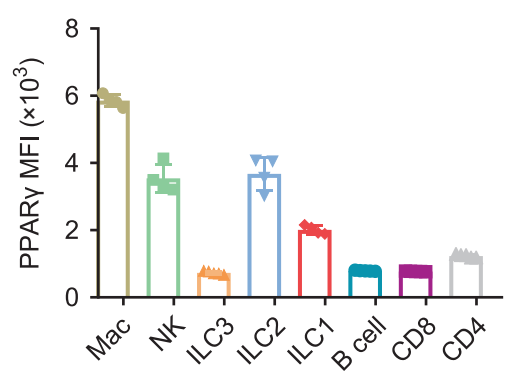

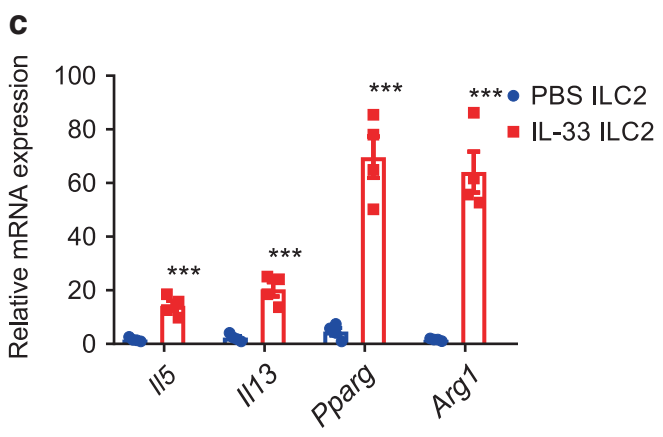

e

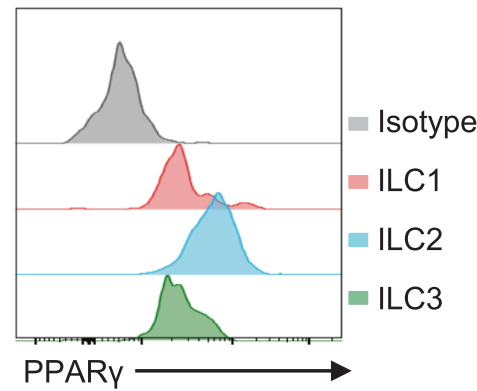

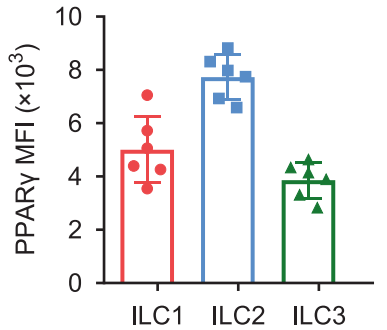

d
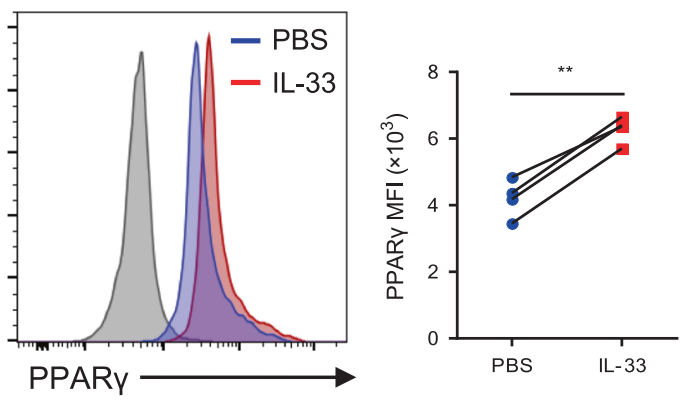

f
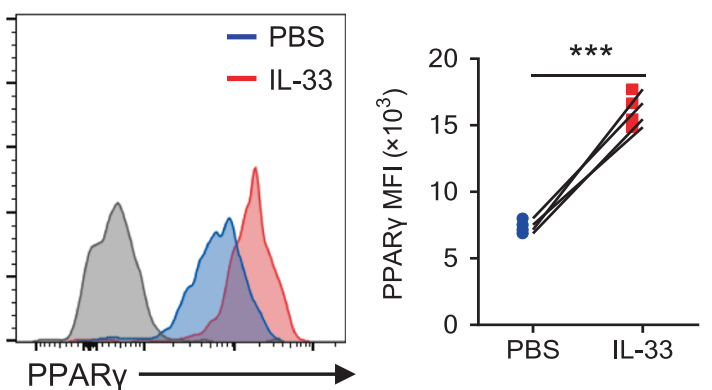

Fig. 1 PPARy is highly expressed in ILC2. a The mRNA expression of PPAR $\gamma$ on macrophage, NK cell, ILC subsets, as well as T and B lymphocytes. b Representative flow cytometric analysis of PPARy expression on immune cells in a. c Lung ILC2s from WT mice were cultured in the presence of IL-2, IL-7 with or without IL-33 for 3 days, the mRNA expression of II5, Il13, Pparg, and Arg1 were analyzed. d Lung cells from WT mice were cultured in the presence of IL-2, IL-7 with or without IL-33 for 3 days, the expression of PPAR $\gamma$ on ILC2 was evaluated by flow cytometry. e Flow analysis of PPAR $\gamma$ expression on human PBMC-derived ILC subsets. $\mathbf{f}$ Human PBMCs were cultured in the presence of IL-2 and IL-7 with or without IL-33 for 3 days, the PPAR $\gamma$ expression was evaluated by flow cytometry. Data are representative of two independent experiments. Error bars show mean \pm SEM; ${ }^{* *} P<0.01 ;{ }^{* *} P<0.001$ by unpaired Student's $t$ test.

which may be cell type dependent. PPARy expression in airway epithelial cells was increased after allergen exposure in sensitized mice. ${ }^{29}$ PPARy agonists reduced cigarette smoke-induced mucin production by epithelial cells, ${ }^{29}$ thus significantly suppressing mucus secretion and collagen deposition. ${ }^{22}$ A lack of PPAR expression in the early stage of hematopoiesis impaired alveolar macrophage (AM) development and caused severe symptoms of influenza virus infection in mice. ${ }^{30}$ On the contrary, dendritic cells treated with PPAR $y$ agonist aggravated airway inflammation after transfer into asthmatic mice, ${ }^{31}$ and specific depletion of PPARy in dendritic cells significantly relieved airway inflammation. ${ }^{32}$ Additionally, PPARY is a positive regulator of Th2 cells during airway inflammation. Th2 cells express higher level of PPARy than other T cell subsets, and type 2 cytokines production in $\mathrm{CD}^{+} \mathrm{T}$ cells strongly depends on PPARy. Mice lacking PPARy expression in T cell fail to develop Th2 cell responses in airway inflammation. ${ }^{32,33}$
These observations collectively indicate that the complicated role of PPARy in lung is highly cell-type dependent.

The potential role of PPARY in ILC2 has recently been indicated. PPARY was found to be highly expressed in ILC2 when compared with other ILC subsets, based on single cell sequencing analysis. ${ }^{34}$ PPARY facilitated lipid uptake and transient lipid storage in lipid droplets, which was required for the fuel of pathogenic ILC2 responses during airway inflammation. ${ }^{35}$ PD-1 has been revealed to be essential for ILC2 function, ${ }^{36}$ while PPARy regulated PD-1 expression on ILC2. ${ }^{37}$ The detailed mechanism of PPARYmediated ILC2 activation, however, remains to be fully explored. In this study, it was found that PPARy deficiency in the hematopoietic system dramatically impaired ILC2 responses to IL-33 or papain challenge, thereby relieving airway inflammation. Further studies showed that IL-33 receptor ST2 mediated the effect of PPARy on ILC2. This study identifies PPARY as an 

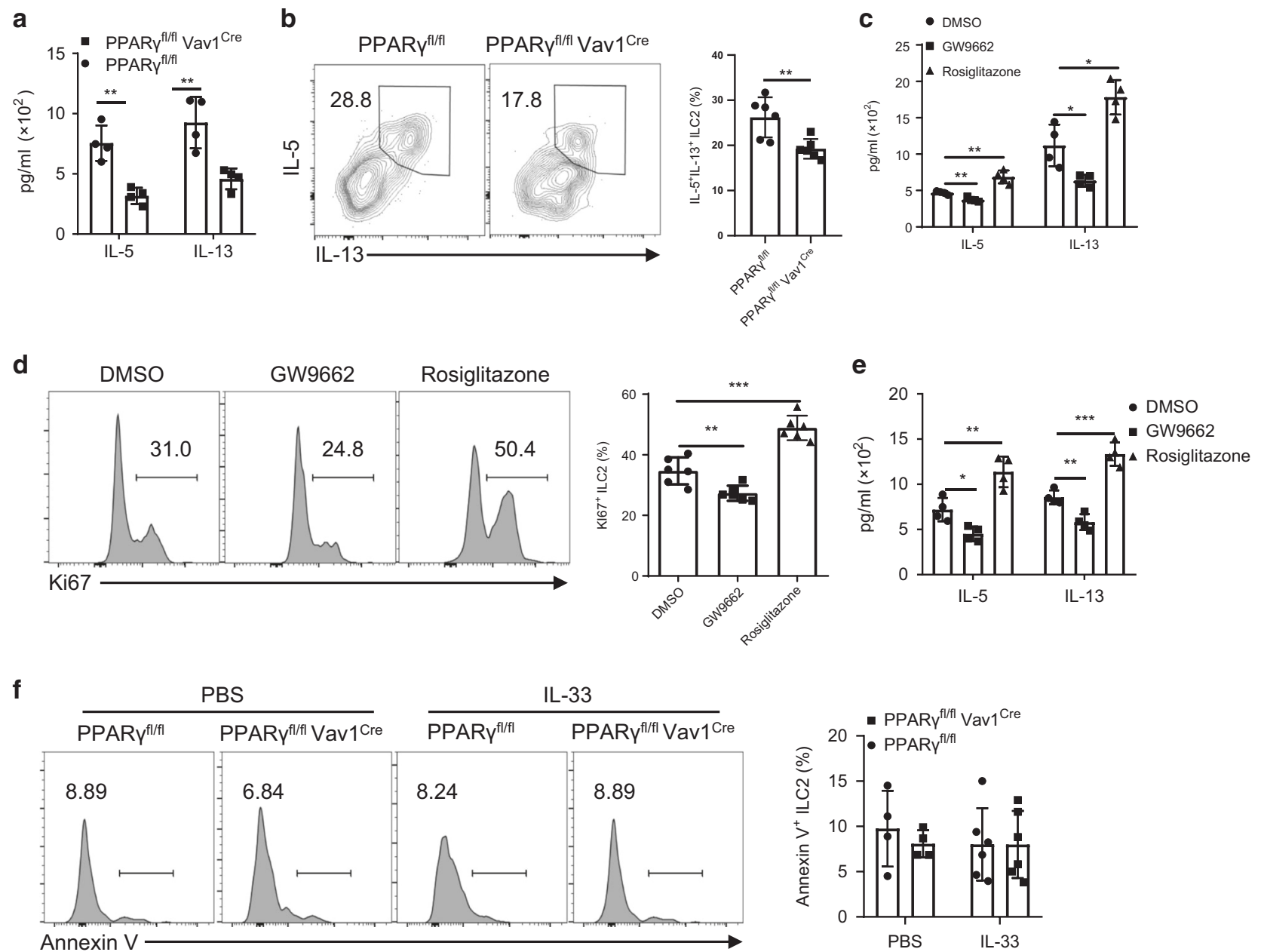

Fig. 2 PPARy enhances ILC2 function in vitro. a Lung ILC2 from PPAR $\gamma^{\mathrm{f} / \mathrm{fl}}$ and PPAR $\gamma^{\mathrm{f} / \mathrm{fl}}$ Vav $1^{\mathrm{Cre}}$ mice were cultured in the presence of IL-2, IL7, and IL-33 for 3 days, IL-5 and IL-13 amount in supernatant were determined by ELISA. b Flow cytometric analysis of IL- $5^{+}$IL-13 $3^{+}$ILC2 of a. c Lung ILC2 from WT mice were cultured in the presence of IL-2, IL-7, and IL-33 for 3 days and treated with rosiglitazone (10 $\mu$ M) or GW9662 (10 $\mu \mathrm{M})$, DMSO was used as control. The amount of IL-5 and IL-13 in supernatant were determined by ELISA. d Ki67 ${ }^{+}$lung ILC2 was analyzed by flow cytometry. e Human PBMC-derived ILC2 were cultured in the presence of IL-2, IL-7, and IL-33 for 3 days and treated with rosiglitazone (10 $\mu \mathrm{M})$ or GW9662 $(10 \mu \mathrm{M})$, DMSO was used as control, IL-5 and IL-13 amount in supernatant were determined by ELISA. $f$ Flow analysis of Annexin $\mathrm{V}^{+}$lung ILC2 levels from PPAR $\gamma^{\mathrm{fl} / \mathrm{fl}}$ and PPAR $\gamma^{\mathrm{f} / \mathrm{fl}} \mathrm{Vav} 1^{\mathrm{Cre}}$ after PBS or IL-33 i.n. challenge. Data are representative of two independent experiments. Error bars show mean $\pm \mathrm{SEM} ;{ }^{*} P<0.05$; ${ }^{* *} P<0.01$; ${ }^{* *} P<0.001$ by unpaired Student's $t$ test.

important regulator of ILC2 in lung, which sheds new light on the role of PPARY in asthma.

\section{RESULTS}

PPARY is dispensable for the development of ILC2

To investigate the potential role of PPARY in ILC2, we first evaluated the expression of PPARy in ILC2 and other types of immune cells. Consistent with previous report, ${ }^{38}$ PPARy was highly expressed in ILC2 $\left(\mathrm{CD} 45^{+} \mathrm{Lin}^{-} \mathrm{CD} 90.2^{+} \mathrm{CD} 25^{+}\right)$both at mRNA and protein levels when compared with those in other ILC subsets and adaptive lymphocytes (Fig. 1a, b). Abundant expression of PPARy in macrophages was observed as expected $^{39}$ (Fig. 1a, b). Challenge with IL-33, the most potent driver of ILC2 activation, 3,40 significantly induced the expression of PPARY and other effector genes in ILC2, including 115, I/13, and Arg1 (Fig. 1c). The induction of PPARy by IL-33 was further evidenced by flow cytometry (Fig. 1d). Similarobservations were noted in human ILC2 $\left(\mathrm{CD} 45^{+} \mathrm{Lin}^{-}\right.$ $\mathrm{CD} 127^{+} \mathrm{CRTH} 2{ }^{+} \mathrm{CD} 161^{+}$), including high expression of PPARy in ILC2 and its upregulation by IL-33 (Fig. 1e, f). The gating strategies of mice and human ILC2 are shown in
Supplementary Fig. S1.,41 These observations indicate the potential importance of PPARY in ILC2 function.

We next asked whether PPARy regulates the homeostasis of ILC2 under steady-state condition. Flow cytometric analysis showed that the expression of PPARy in the progenitors of ILC2, including CLP, aLP, and ChILP, was considerably lower when compared with that in immature ILC2 from bone marrow (Supplementary Fig. S2a, b). Next, PPARy was specifically deleted in the hematopoietic system by cross-breeding the $V a v{ }^{1}{ }^{C r e}$ strain with $P P A R \gamma^{\mathrm{fl} / \mathrm{fl}}$ mice (termed PPAR $\gamma^{\mathrm{fl} / \mathrm{fl}}$ Vav ${ }^{\mathrm{Cre}}$ thereafter). Flow cytometric analysis showed that the frequencies of distinct ILC2 progenitors did not differ between PPAR $\gamma^{\mathrm{f} / \mathrm{fl}}$ Vav $1^{\mathrm{Cre}}$ mice and PPAR $\gamma^{\mathrm{f} / \mathrm{fl}}$ controls (Supplementary Fig. S2c), thereby indicating that the development of ILC2 was not affected by PPARY. Furthermore, the numbers of ILC2 in the peripheral tissues, including lung, visceral adipose tissue (VAT), and lamina propria from the large intestine (LI), were comparable between PPAR $\gamma^{\text {fl/fl }}$ $\mathrm{Vav}_{1}{ }^{\mathrm{Cre}}$ and PPAR ${ }^{\mathrm{fl} / \mathrm{fl}}$ mice (Supplementary Fig. S2d). Other ILC subsets, including NK cell in the spleen, ILC1 in the mesenteric lymph node $(\mathrm{mLN})$ and large intestine (Supplementary Fig. S2e), and ILC3 in mLN and large intestine (Supplementary Fig. S2f, g), were not apparently affected by PPARy deficiency. These 
a
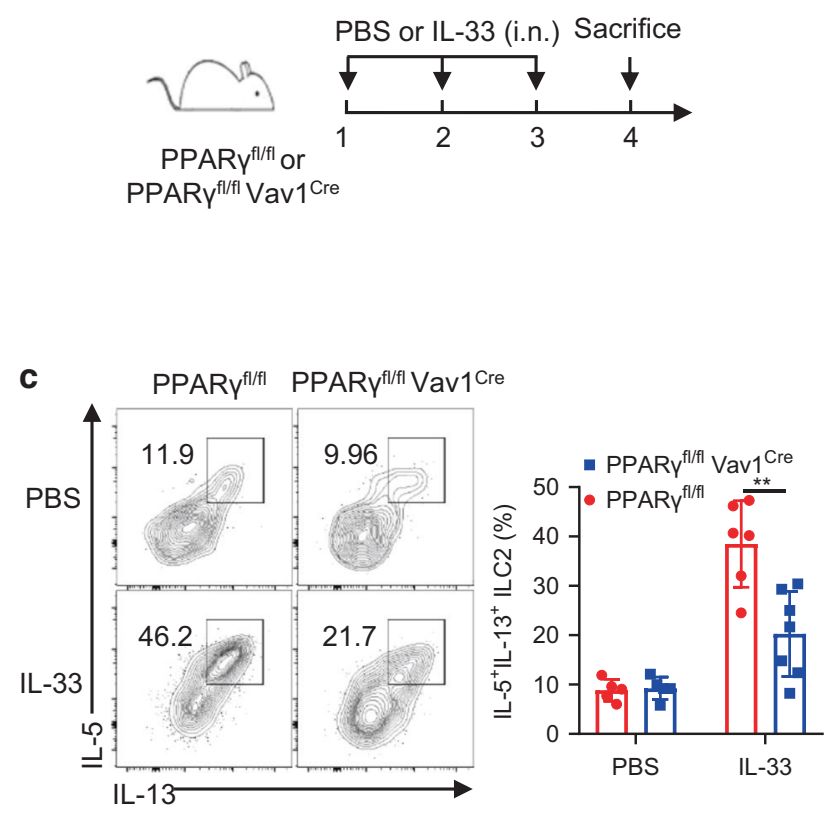

e

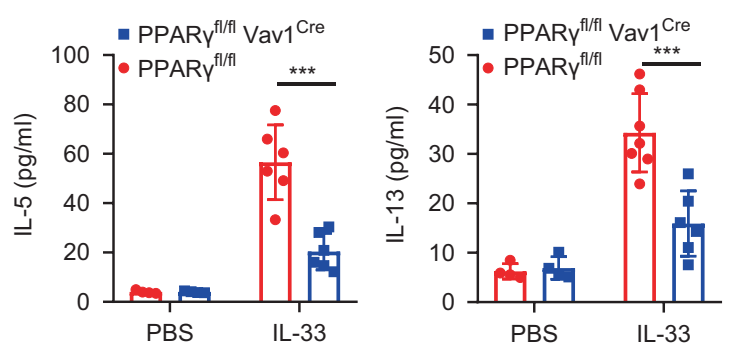

g

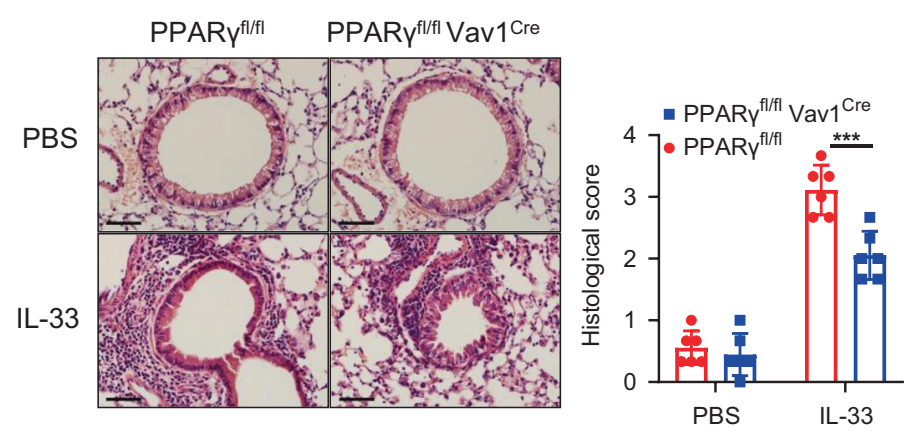

b
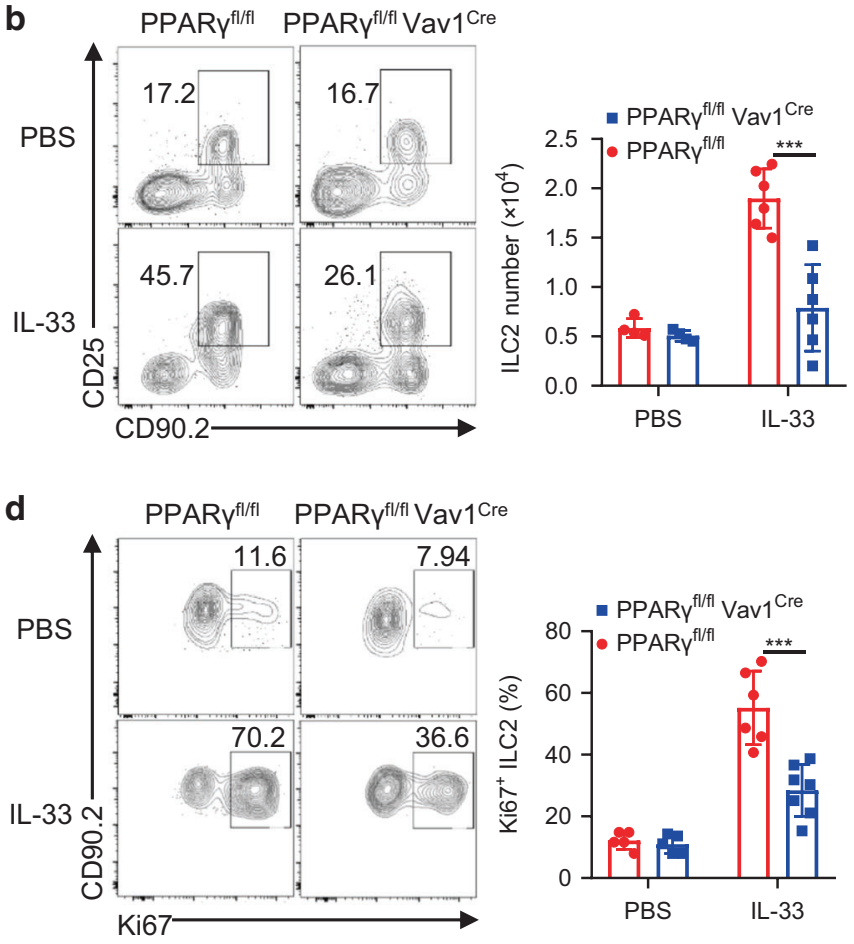

f
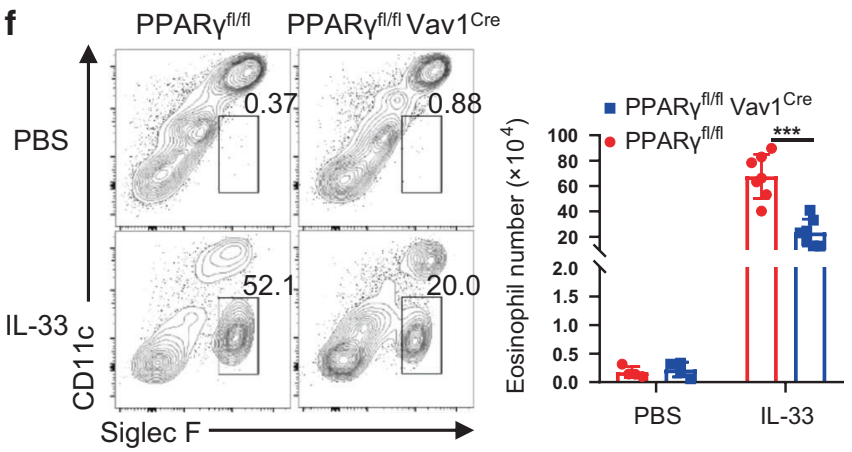

Fig. 3 PPARy deficiency impairs ILC2 responses to IL-33 in vivo. a PPAR $\gamma^{\mathrm{f} / \mathrm{fl}}$ and PPAR $\gamma^{\mathrm{f} / \mathrm{fl}}$ Vav1 ${ }^{\text {Cre }}$ mice were intranasally treated with IL-33 ( $500 \mathrm{ng} /$ mouse/day) or PBS for 3 consecutive days, mice were sacrificed $24 \mathrm{~h}$ after last challenge. b Flow cytometric analysis of lung ILC2

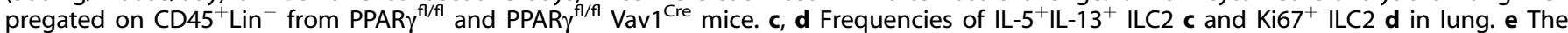
amount of IL-5 and IL-13 in BALF was examined by ELISA. f Eosinophils (CD45 ${ }^{+}$CD $11 c^{-}$Siglec- $F^{+}$) levels in BALF were analyzed by flow cytometry. $\mathbf{g}$ H\&E staining of lung tissues (bars, $100 \mu \mathrm{m})$. Data are representative of two independent experiments $(n=4-6 \mathrm{for}$ PBS groups, $n$ $=6$ for IL-33 groups). Error bars show mean $\pm \mathrm{SEM} ;{ }^{* *} P<0.01 ;{ }^{* * *} P<0.001$ by unpaired Student's $t$ test.

observations indicate that PPARy is dispensable for the development of ILC2 and other ILC subsets.

PPAR $y$ enhances ILC2 function both in vitro and in vivo To study the role of PPARY in the biological function of ILC2, lung ILC2s were sorted and cultured in the presence of IL-2, IL-7, and IL-
33 for 3 days in vitro. The amounts of IL-5 and IL-13 in the supernatants were determined by ELISA. Results showed that ILC2 from PPAR $\gamma^{\text {fl/fl }}$ Vav $1{ }^{\text {Cre }}$ mice produced remarkably lower level of

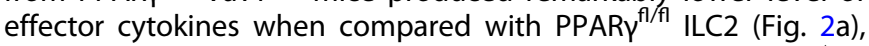
which were further confirmed by the proportions of $\mathrm{IL}-5^{+} \mathrm{IL}-$ $13^{+}$ILC2 (Fig. 2b). In line with these observations, PPARy agonist 
a
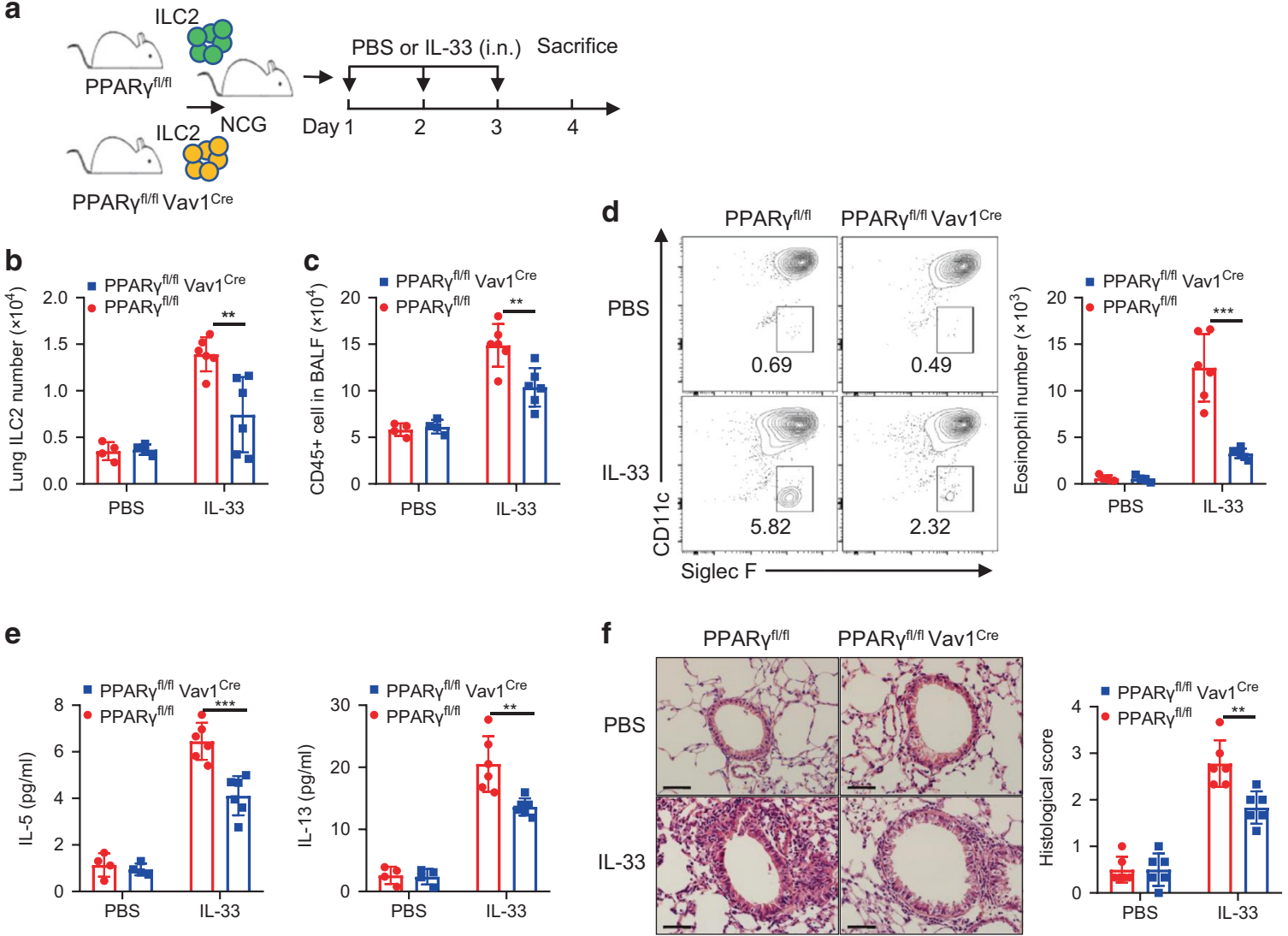

Fig. 4 PPARy-deficient ILC2 fails to induce lung inflammation. a PPAR $\gamma^{\mathrm{fl} / \mathrm{fl}}$ or PPAR $\gamma^{\mathrm{fl} / \mathrm{fl}}$ Vav $1^{\mathrm{Cre}}$ mice-derived lung ILC2 was sorted and transferred into NCG mice, respectively, followed by IL-33 or PBS i.n. challenge for 3 consecutive days, mice were sacrificed $24 \mathrm{~h}$ after last challenge. $\mathbf{b}$ The number of ILC2 in lung of recipient mice. $\mathbf{c}$ The number of total CD45 ${ }^{+}$cells in BALF. d The frequencies and number of eosinophils in BALF determined by flow cytometry. e The amount of IL-5 and IL-13 in BALF was examined by ELISA. $\mathbf{f}$ H\&E staining of lung tissues (bars, $100 \mu \mathrm{m}$ ). Data are representative of two independent experiments $(n=4-6$ for PBS groups, $n=6$ for IL-33 groups). Error bars show mean $\pm \mathrm{SEM} ;{ }^{* *} P<0.01 ;{ }^{* * *} P<0.001$ by unpaired Student's $t$ test.

rosiglitazone enhanced the cytokines production and cell proliferation of ILC2, whereas PPARY antagonist GW9662 displayed opposite effects (Fig. 2c, d). Similar results were obtained for human ILC2 (Fig. 2e). PPARy deficiency did not affect the apoptosis of ILC2 under steady and IL-33 stimuli situation (Fig. 2f). These observations indicate that PPARY is a positive regulator of ILC2.

We next explored whether PPARy affects the function of ILC2 by evaluation of their responses to IL-33 challenge in vivo. PPAR $\gamma^{\mathrm{fl} / \mathrm{fl}}$ and PPAR $\gamma^{\mathrm{f} / \mathrm{fl}}$ Vav1 ${ }^{\mathrm{Cre}}$ mice were treated with IL-33 intranasally for 3 consecutive days (Fig. 3a). As expected, the numbers of lung ILC2 were dramatically increased after intranasal administration of $\mathrm{IL}-33$ in both PPAR $\gamma^{\mathrm{fl} / \mathrm{fl}} \operatorname{Vav}^{\mathrm{Cre}}$ and $\mathrm{PPAR}^{\mathrm{fl} / \mathrm{fl}}$ mice (Fig. 3b), whereas the induction fold was significantly reduced in PPAR $\gamma^{\text {fl/fl }}$ $\mathrm{Vav}^{\mathrm{Cre}}$ mice when compared with that in PPARY ${ }^{\text {flf }}$ littermates (Fig. 3b). Production of the effector cytokines IL-5 and IL-13 by lung ILC2, as well as proliferation of ILC2, were clearly diminished in PPAR $\gamma^{\mathrm{f} / \mathrm{fl}}$ Vav ${ }^{\mathrm{Cre}}$ mice (Fig. 3c, d). Furthermore, the amounts of IL-5 and IL-13, as well as eosinophilic infiltration, in bronchoalveolar lavage fluid (BALF) were apparently reduced in $P P A R \gamma^{f / f l}$ $V a v 1^{C r e}$ mice when compared with those in PPAR $\gamma^{\text {fl/fl }}$ littermates (Fig. 3e, f). The attenuation of lung inflammation in PPARy ${ }^{\mathrm{fl} / \mathrm{fl}}$ $\mathrm{Vav} 1^{\mathrm{Cre}}$ mice was further evidenced by $\mathrm{H} \& \mathrm{E}$ histological staining (Fig. $3 \mathrm{~g}$ ). These results suggested that PPARY deletion impairs ILC2 responses to IL-33 in the lung.

For further confirmation, adoptive transfer of ILC2 was performed. Equal numbers of lung ILC2 from PPAR $\gamma^{\mathrm{f} / \mathrm{fl}}$ and $\mathrm{PPAR}^{\mathrm{fl} / \mathrm{fl}}$ Vav1 ${ }^{\mathrm{Cre}}$ mice were adoptively transferred into immune- deficient NCG mice, followed by intranasal administration of IL-33 for 3 consecutive days (Fig. 4a). Results showed that the numbers of lung ILC2 in the recipients were significantly increased after IL33 administration when compared with that in PBS control group (Fig. 4b), whereas the fold induction in recipients of PPARy ${ }^{\text {fl/f }}$ $\mathrm{Vav}_{1}{ }^{\mathrm{Cre}}$ ILC2s was significantly lower than that in PPAR $\gamma^{\mathrm{f} / \mathrm{fl}}$ control (Fig. 4b). Consequently, the total number of $\mathrm{CD}_{4} 5^{+}$cells, the number of infiltrated eosinophils in BALF, and the amounts of IL-5 and IL-13 in BALF, were consistently lower in recipients of PPAR $\gamma^{\mathrm{f} / \mathrm{fl}}$ Vav1 ${ }^{\text {Cre }}$ ILC2 (Fig. 4c-e). The attenuation of lung inflammation was further evidenced by H\&E staining (Fig. 4f). Collectively, these observations support that PPARY deficiency intrinsically impairs ILC2 function.

In addition to IL-33, IL-25 is another strong inducer of ILC2s. ILC2s induced by IL-25 are termed inflammatory ILC2s (ilLC2s). ${ }^{42}$ PPAR $\gamma^{\mathrm{f} / \mathrm{fl}}$ and PPAR $\gamma^{\text {fl/fl }} \mathrm{Vav}^{\mathrm{Cre}}$ mice were intraperitoneally (i.p.) injected with recombinant IL-25 for 3 days, the level of inflammatory ILC2 ( Lin $^{-} \mathrm{ST}^{-} \mathrm{KLRG}^{\mathrm{hi}}$ ) in lung was significantly induced in both PPAR $\gamma^{\text {fl/fl }}$ Vav $1^{\text {Cre }}$ and control mice, whereas there was no differences between them (Supplementary Fig. S3a). The production of effector cytokines IL-4 and IL-13 by ilLC2s also failed to display any noticeable differences between PPAR ${ }^{\mathrm{fl} / \mathrm{fl}}$ Vav1 ${ }^{\mathrm{Cre}}$ and PPAR $\gamma^{\mathrm{f} / \mathrm{fl}}$ mice (Supplementary Fig. S3b). These observations indicate that deletion of PPARY may not affect IL-25-induced ilLC2s.

Although PPARy did not affect the production of other ILC subsets, we further investigated whether PPARy deletion affects the functions of ILC1 and ILC3 in a Citrobacter rodentium infection- 
a
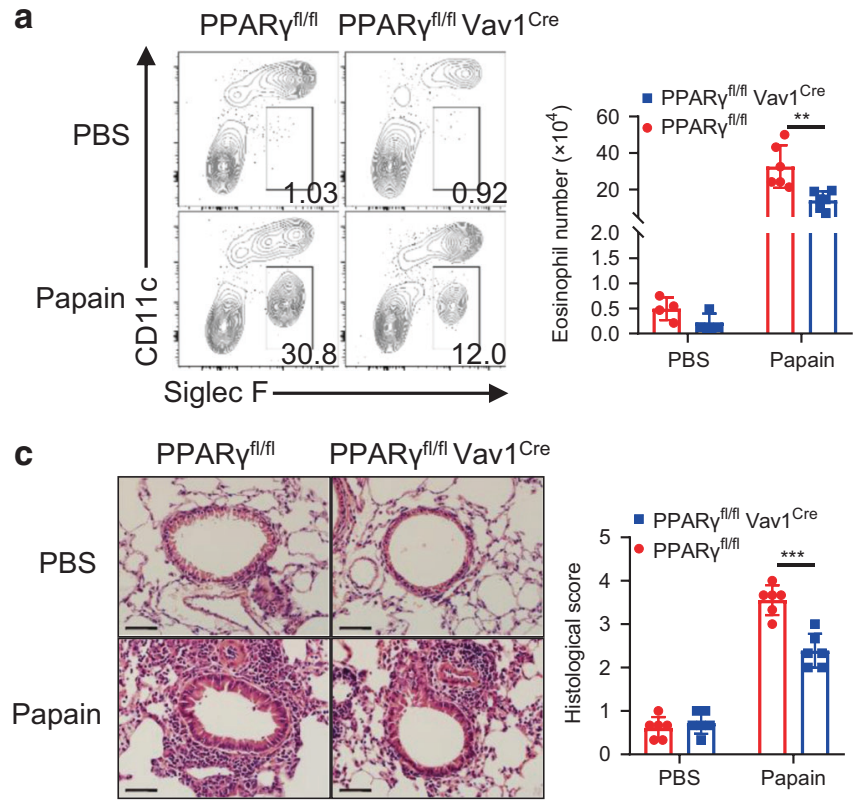

e
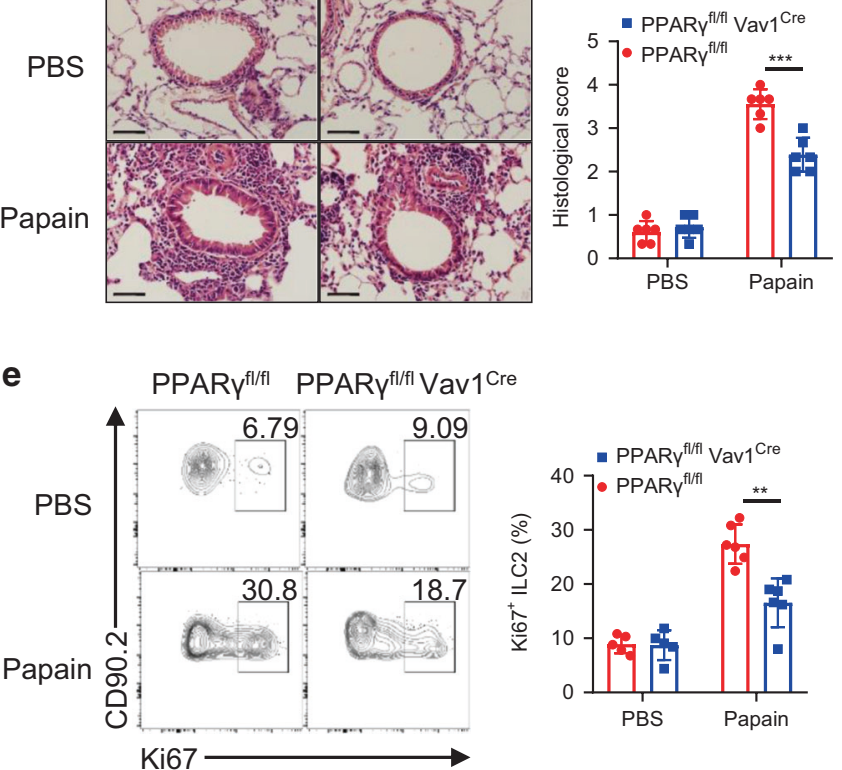

g

Anti-CD4 antibody i.p.

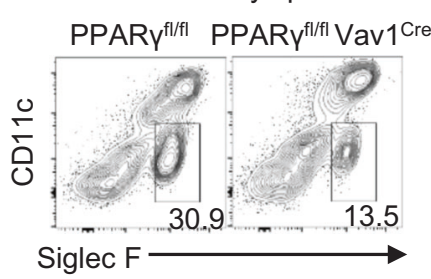

i
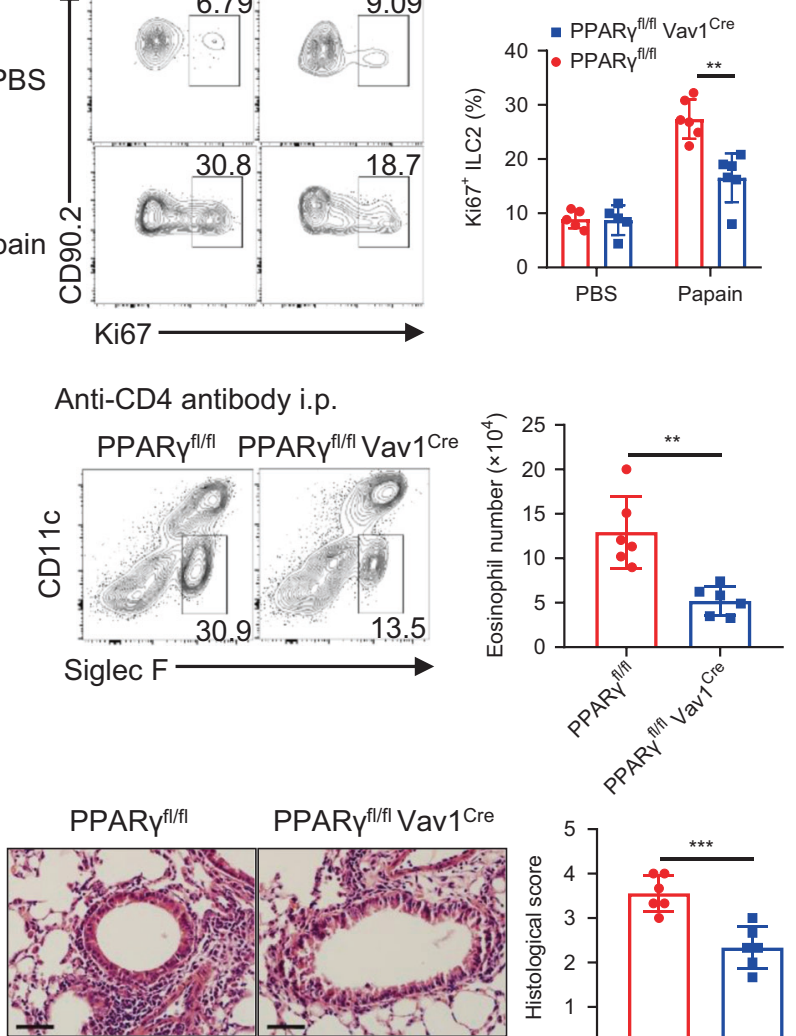

b
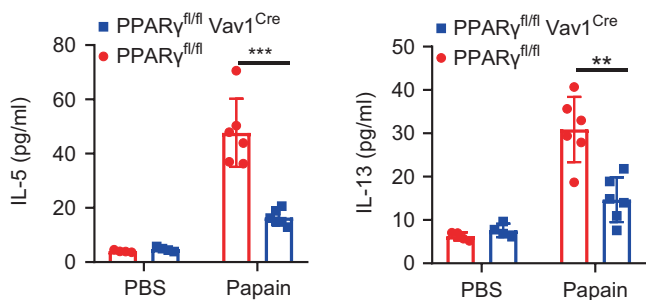

d

PPARYfl/fl PPAR $y^{\mathrm{fl} / f l}$ Vav1Cre
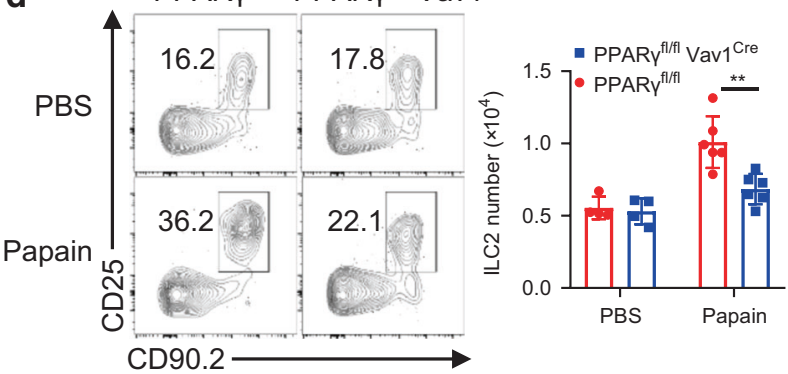

f
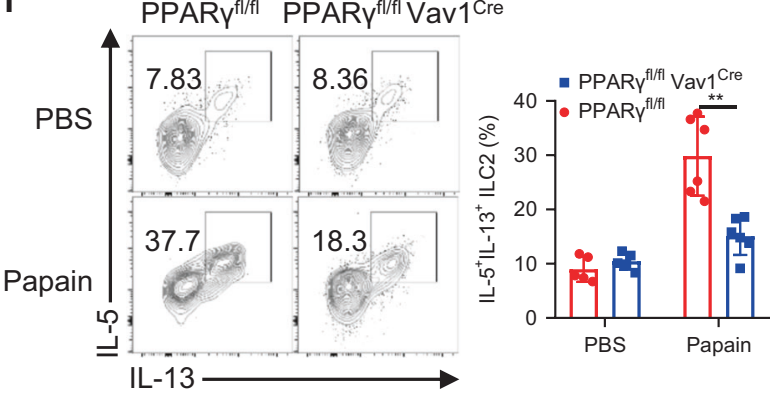

h
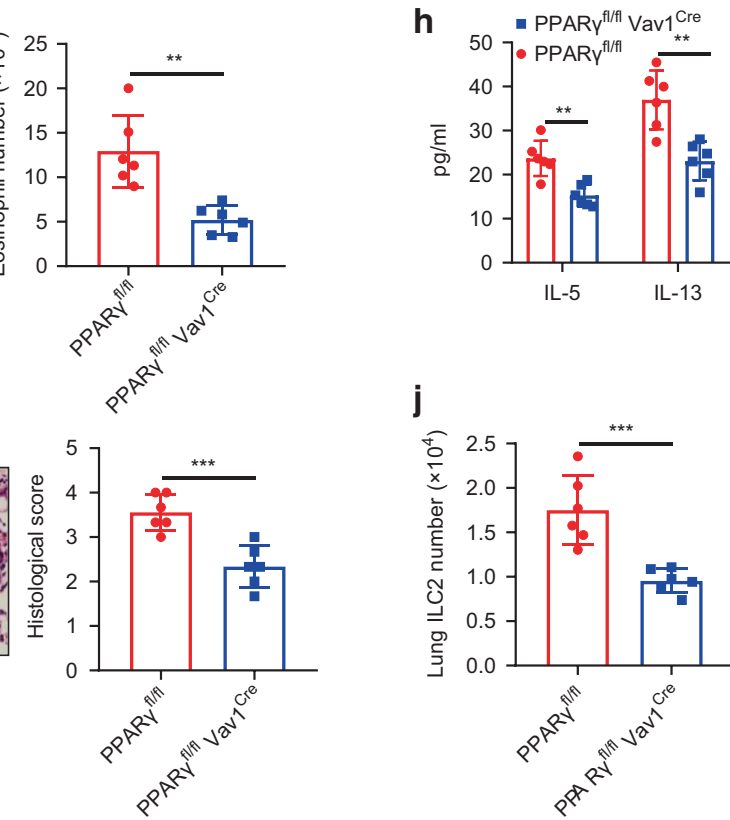

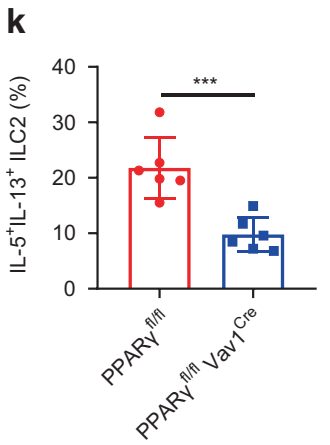

induced murine colitis model. ${ }^{43,44}$ PPAR $^{\mathrm{fl} / \mathrm{fl}}$ and PPAR $\gamma^{\mathrm{f} / \mathrm{fl}}$ Vav $1{ }^{\mathrm{Cre}}$ mice were orally gavaged with $2 \times 10^{9}$ colony-forming units (CFUs) of $C$. rodentium and sacrificed on day 6 . The results revealed comparable colitis symptoms between PPAR $\gamma^{\text {fl/fl }}$ and PPAR $\gamma^{\mathrm{fl} / \mathrm{fl}}$
$\mathrm{Vav} 1^{\mathrm{Cre}}$ mice, as indicated by similar weight loss and shortened colon length (Supplementary Fig. S4a, b). The production of interferon (IFN)- $\gamma$ by ILC1, production of IL-17, IL-22, and IFN- $\gamma$ by ILC3 in colon, displayed no differences between PPARY $\gamma^{\mathrm{fl} / \mathrm{fl}}$ and 


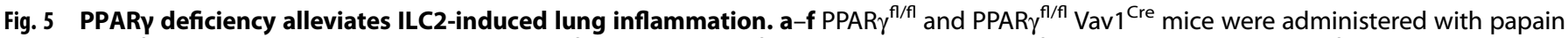

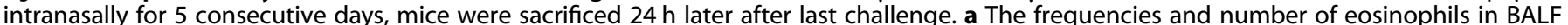

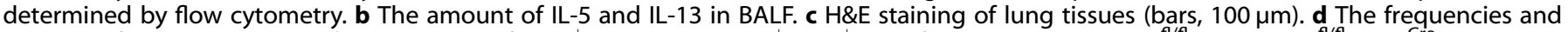

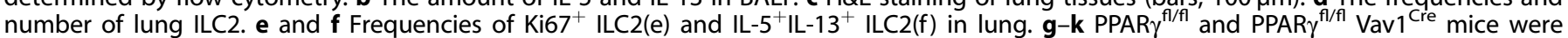

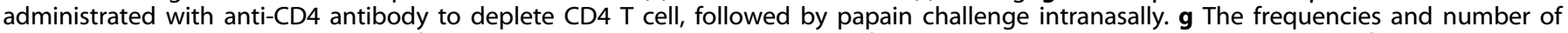

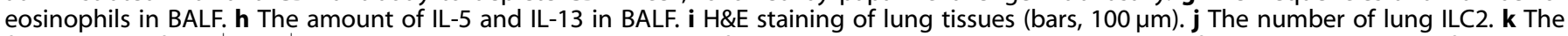

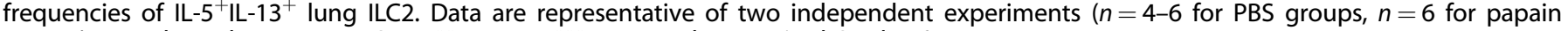
groups). Error bars show mean $\pm \mathrm{SEM}$; ${ }^{* *} P<0.01$; ${ }^{* *} P<0.001$ by unpaired Student's $t$ test.

PPARY $\gamma^{\mathrm{f} / \mathrm{fl}}$ Vav1 ${ }^{\mathrm{Cre}}$ mice (Supplementary Fig. S4c, d). These observations suggest that deletion of PPARy did not affect the function of other ILC subsets.

\section{PPARy aggravates ILC2-mediated lung inflammation}

Considering the importance of ILC2 in the pathogenesis of airway inflammation, ${ }^{45}$ we next evaluated the effect of PPARy deficiency on ILC2-induced lung inflammation. Intranasal instillation of papain for 5 consecutive days was performed. The numbers of infiltrated eosinophils, as well as the amounts of type 2 cytokines in BALF, were clearly diminished in PPAR $\gamma^{\mathrm{f} / \mathrm{fl}} \mathrm{Vav} 1^{\text {Cre }}$ mice as compared with PPAR $\gamma^{\mathrm{fl} / \mathrm{fl}}$ control upon papain challenge (Fig. 5a, b). The alleviation of lung inflammation in PPARy ${ }^{\mathrm{fl} / \mathrm{fl}}$ Vav1 $1^{\mathrm{Cre}}$ mice was further supported by $\mathrm{H} \& \mathrm{E}$ staining (Fig. $5 \mathrm{C}$ ). As expected, the frequencies and absolute numbers of lung ILC2 in PPAR $\gamma^{\mathrm{fl} / \mathrm{fl}}$ Vav $1^{\text {Cre }}$ mice were significantly lower than those in PPAR $\gamma^{\mathrm{fl} / \mathrm{fl}}$ mice (Fig. $5 \mathrm{~d}$ ). The cell proliferation and cytokine production of lung ILC2 were consistently decreased in PPARY ${ }^{\mathrm{fl} / \mathrm{fl}}$ Vav ${ }^{\mathrm{Cre}}$ mice (Fig. 5e, f). As further confirmation, Alternaria alternata, a clinically relevant allergen, was used to induce lung inflammation. In line with the observations from papain model, the tissue inflammation in lung (Supplementary Fig. S5a, b, c) and the responses of ILC2 in lung (Supplementary Fig. S5d, e, f) were dramatically reduced in PPAR $\gamma^{\text {fl/fl }}$ Vav $1^{\text {Cre }}$ mice. These observations suggested that PPARY aggravates ILC2-driven lung inflammation.

PPARY is essential for the function of Th2 cells, ${ }^{32,33}$ and there is mutual regulation between Th2 and ILC2. ${ }^{6,46,47}$ To determine whether the effect of PPARy on ILC2 was dependent or independent of CD4 T cells, PPAR $\gamma^{\mathrm{f} / \mathrm{fl}}$ and PPAR $\gamma^{\mathrm{fl} / \mathrm{fl}}$ Vav $1^{\mathrm{Cre}}$ mice were treated with anti-CD4 antibody to deplete CD4 ${ }^{+} \mathrm{T}$ cells ${ }^{48}$ and followed by intranasal instillation of papain for 5 consecutive days (Supplementary Fig. S5g). Results showed that PPAR $\mathrm{f}^{\mathrm{fl} / \mathrm{fl}}$ Vav1 ${ }^{\mathrm{Cre}}$ mice consistently exhibited lower levels of eosinophils in BALF (Fig. 5g), reduced amounts of IL-5 and IL-13 in BALF (Fig. 5h), as well as attenuation of lung inflammation (Fig. 5i) upon depletion of $\mathrm{CD}^{+} \mathrm{T}$ cells. The numbers of ILC2 in lung and their secretion of IL-5 and IL-13 in PPAR $\gamma^{\text {fl/fl }}$ Vav $1^{\text {Cre }}$ mice were reduced as expected (Fig. 5j, k). These results indicated that the regulation of ILC2 by PPARY was independent of CD4 T cells.

\section{ST2 mediates the effects of PPARy on ILC2}

We next explored the mechanisms underlying the effects of PPARY on ILC2. The level of GATA3, the master regulator of ILC2, was not affected by the presence or absence of PPARY (Fig. 6a). However, the expression of IL-33 receptor ST2 was significantly downregulated in ILC2 upon PPARy deletion (Fig. 6b). In addition, RT-PCR analysis confirmed the downregulation of ST2 at the transcriptional level (Fig. 6c). Furthermore, lung ILC2 from wild type mice were sorted and cultured with IL-2, IL-7, and IL-33 in the presence of PPARy agonist rosiglitazone or antagonist GW9662 for 3 days. Results showed that the expression of ST2 was significantly upregulated by rosiglitazone, whereas it was decreased by GW9662 when compared with DMSO control (Fig. 6d).

Promoter analysis revealed several potential binding sites of PPARY in the regulatory region of il1rl1 that encodes ST2 (Fig. 6e). Further ChIP assay showed that PPARy protein could bind with the locus of site 3 on the il1rl1 promoter, aP2 promoter region with known PPARy-binding site was used as a positive control, while a region in il1rl1 promoter without predicted PPARy-binding site (il1rl1 non) was used as a negative control ${ }^{49,50}$ (Fig. 6f). These results identified il1rl1 as a downstream transcriptional target of PPARy in ILC2. Next, ST2 was overexpressed in PPARy ${ }^{\mathrm{fl} / \mathrm{fl}}$ Vav1 ${ }^{\mathrm{Cre}}$ ILC2 by retroviral infection, the mRNA expression of ST2 in PPARgdeficient ILC2 was dramatically upregulated to wild type level after retroviral infection (Supplementary Fig. S6a, b). Importantly, ST2 overexpression almost completely rescued the defective production of IL-5 and IL-13 in PPARY ${ }^{\mathrm{f} / \mathrm{fl}}$ Vav1 ${ }^{\text {Cre }}$ ILC2 (Fig. 6g), suggesting that ST2 mediated the effect of PPARY on ILC2 function.

\section{DISCUSSION}

The importance of PPAR in airway inflammation has been appreciated, although conflicting results have been reported from different groups. Clinical trials of asthma treatment by PPARY agonists showed minor or even negligible effects. ${ }^{51-53}$ These reports indicate the complicated role of PPARy in lung. Here, we reported that PPARY is an important regulator of ILC2 via its downstream target ST2. Deficiency of PPARY in hematopoietic system impaired ILC2 response in lung, thus alleviating ILC2induced airway inflammation.

In addition to its crucial role in lipid metabolism, the importance of PPAR $y$ in the regulation of immunity and tissue inflammation has been well documented. ${ }^{21}$ PPARy may function as a bridge to link metabolism and immunity. Karagiannis et al. ${ }^{35}$ recently demonstrated that IL-33 promotes FA uptake via induction of PPARy, which provides fuel for the proliferation of pathogenic ILC2 responses. In our study, we found that PPARy upregulate IL-33 receptor ST2 expression on ILC2. It is therefore possible that there exists a positive feedback loop between PPARy and IL-33-ST2 signaling in the regulation of ILC2 activation. Whereas there is no evidence that PPARy regulates FA uptake via ST2. Another earlier research ${ }^{54}$ by the same author from Immunity 2020 reported that FA uptake and metabolism are essential for intestinal ILC2 function and ILC2-mediated resistance to helminth infection, in which they showed that pparg as well as other genes regulate FA usage in small intestine (SI) Lin Thy $1.2^{+} \mathrm{KLRG} 1^{+}$ILC2. As we know, Lin Thy $1.2^{+} \mathrm{KLRG}^{+}$ILC2 in SI under helminth infection was defined as ilLC2 that lack ST2 expression. ${ }^{42}$ These reports indicate that PPARY controls FA uptake of ILC2 in the absence of ST2 in ilLC2. Furthermore, PPARY regulates FA metabolism in multiple organs, while most of them do not express ST2, ${ }^{55}$ indicated that ST2 is not necessary for PPARY-mediated FA uptake. Based on these literatures, whether there is cross-talk between FA uptake and ST2 induction in the regulation of ILC2 by PPARY deserves to be further investigated.

The effects of PPARy on airway inflammation were reported by different groups, $^{30-33}$ although the observations were not consistent with each other. The possible explanations underlying these contraries may be caused by different animal models or different ways to modulate the expression or activity of PPAR $\gamma$. PPARy is expressed in distinct cell types. Administration of PPARY agonist or antagonists to animals may display different effects which may be dependent on cell type or tissue, utilization of conditional knock-out of PPARy in specific cell types is therefore 
a

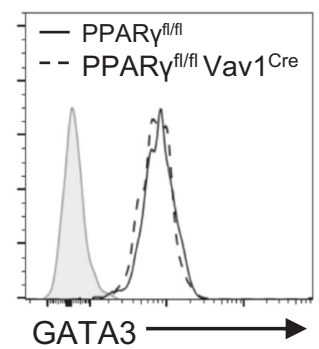

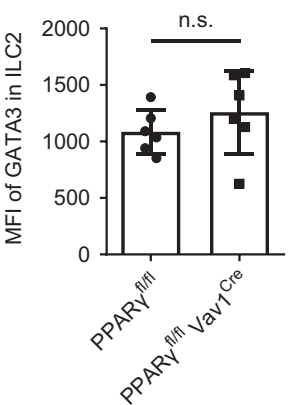

b
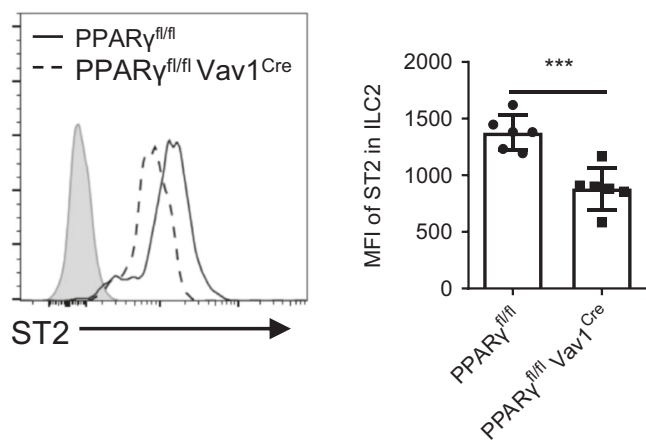

C

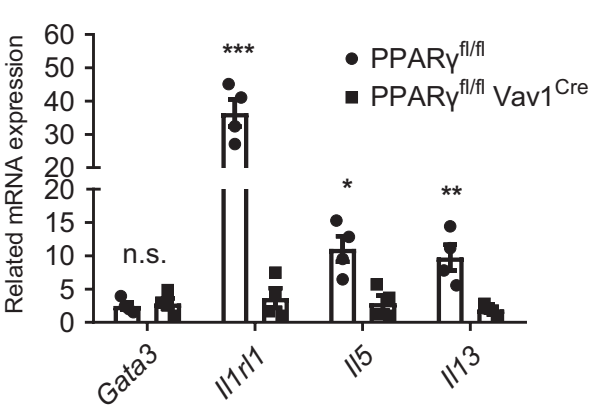

d

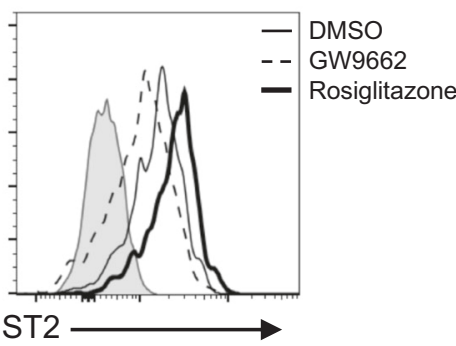

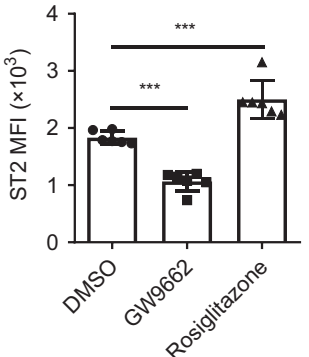

e

PPARy binding site $=$

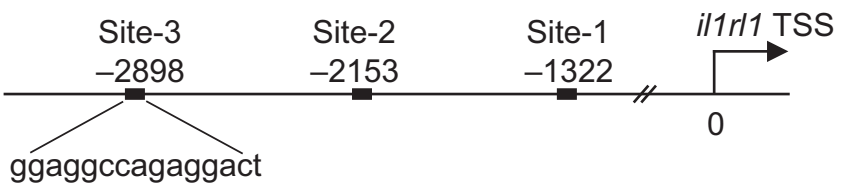

f

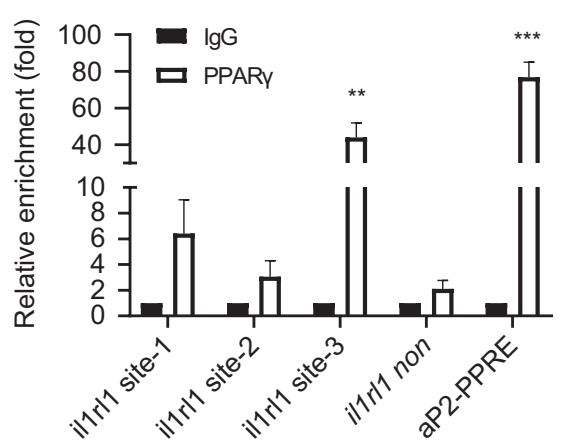

g

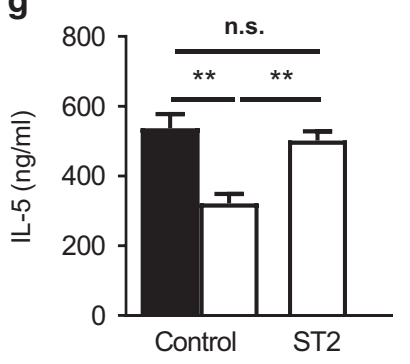

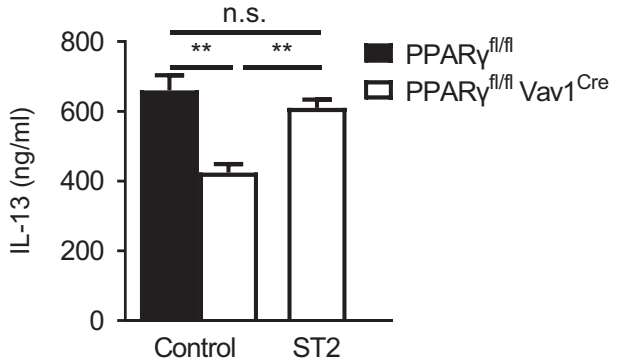

Fig. 6 ST2 mediates the effect of PPARy on ILC2. $\mathbf{a}$ and $\mathbf{b}$ Flow cytometric analysis of GATA3 a and ST2 b expression in lung ILC2 from

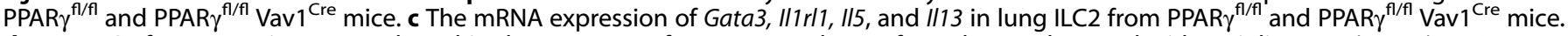
d Lung ILC2 from WT mice were cultured in the presence of IL-2, IL-7, and IL-33 for 3 days and treated with rosiglitazone (10 $\mu$ M) or GW9662 $(10 \mu \mathrm{M})$, DMSO was used as control. MFI of ST2 were measured in lung ILC2. e Three potential PPAR $\gamma$-binding sites in il1rl1 promotor region. $\mathbf{f}$ ChIP assay of PPAR $\gamma$ binding on the $/ 11$ rl1 promotor. A region of aP2 promoter with known PPARg-binding site was chosen as positive control (aP2-PPRE), a region in I/1rl1 promoter, where PPARg is not expected to bound was included as negative control (I/1rl1 non). g Lung ILC2 from PPAR $\gamma^{\mathrm{f} / \mathrm{fl}}$ and PPAR $\gamma^{\mathrm{f} / \mathrm{fl}}$ Vav ${ }^{\mathrm{Cre}}$ mice were infected with retrovirus overexpression of ST2 or vector control in the presence of IL-2, IL-7, and IL33 for 3 days. The amount of IL- 5 and IL-13 in supernatants were measured by ELISA. Data are representative of two independent experiments $(n=6)$. Error bars show mean \pm SEM; n.s., not significant; ${ }^{*} P<0.05 ;{ }^{* *} P<0.01 ;{ }^{* * *} P<0.001$ by unpaired Student's $t$ test a-f or one-way ANOVA with Bonferroni post-test $\mathbf{g}$.

helpful to address its complicated roles. In this study, conditional knock-out of PPARy in the hematopoietic system was conducted to minimize its effect on non-hematopoietic cells. In order to exclude the potential effects of T cells, B cells, NK cells, and myeloid cells, immunodeficient NCG mice were used. Adoptive transfer of ILC2 from PPAR ${ }^{\mathrm{f} / \mathrm{fl}} \operatorname{Vav} 1^{\text {Cre }}$ mice displayed lower capability to induce lung inflammation in recipient mice. Antibody depletion of CD4 T cells was also conducted to further exclude the potential effect of T lymphocytes, since PPARy was reported to regulate Th2 cells. ${ }^{32,33}$ The in vitro ILC2 culture system further indicate the direct effect of PPARY on ILC2. These observations collectively support that the regulation of ILC2 by PPARY is cell intrinsic and independent of other cell types.

PPAR $y$ is a master regulator in adipose tissue by regulating adipogenesis. ILC2 in fat tissue play an important role in lipogenesis. Min-Woo et al. first reported that activated ILC2 
476

promotes the proliferation of bipotential adipocyte precursors and enhances their commitment to beige fat lineage, which was dependent on IL-33. ${ }^{56}$ Meanwhile, Jonathan et al. reported ILC2 in white adipose tissue could promote beiging of adipose and limit the development of obesity by production of enkephalin peptides. ${ }^{12}$ Whether the deficiency of PPARY affects the function of ILC2 in fat tissue deserves further investigation.

In conclusion, this study demonstrated PPARy as an important regulator of ILC2 during allergic inflammation. Downregulation of ST2 on the surface of ILC2 may explain the defective ILC2 responses upon PPARy deletion. This study indicates the complicated role of PPARY in lung homeostasis.

\section{METHODS}

Mice

$\mathrm{PPAR}^{\mathrm{fl} / \mathrm{fl}}$ mice (B6.129-Ppargtm2Rev/J, 004584) were kindly provided by Dr. Shengzhong Duan from the Shanghai Ninth People's Hospital, College of Stomatology, Shanghai Jiao Tong University School of Medicine. Vav1 ${ }^{\text {Cre }}$ mice (B6.Cg-Commd10Tg (Vav1-icre)A2Kio/J, 008610) were purchased from The Jackson Laboratory. NCG mice (NOD-Prkdc ${ }^{\mathrm{em} 26 \mathrm{Cd} 52} \| 2 \mathrm{rg}^{\mathrm{em} 26 \mathrm{Cd} 22} / \mathrm{Nju}$ ) were purchased from Nanjing Biomedical Research Institute of Nanjing University (Nanjing, China). C57BL/6 mice were purchased from Guangdong Medical Laboratory Animal Center (Guangzhou, China). All mice were used at the age of 6-8 weeks. All animal experiments were approved by the Institutional Animal Care and Use Committee of Tianjin Medical University and Sun Yat-sen University.

Murine airway inflammation models

For IL-33-induced model, ${ }^{57}$ mice were intranasally treated with recombinant mouse IL-33 (500 ng/mouse/day; BioLegend) or PBS for 3 consecutive days and sacrificed $24 \mathrm{~h}$ later after the final treatment. For papain-induced type 2 airway inflammation, ${ }^{58} 20$ $\mu \mathrm{g}$ papain (in $40 \mu \mathrm{l} \mathrm{PBS} /$ mouse/day, sigma) or PBS was intranasally administrated to mice for 5 consecutive days, BALF and lung samples were analyzed $24 \mathrm{~h}$ after last challenge. For Alternaria alternata-induced lung inflammation, ${ }^{57}$ mice were intranasally treated with Alternaria alternata $(100 \mu \mathrm{g}$ in $40 \mu \mathrm{l} \mathrm{PBS} /$ mouse/ day, Greerlabs) for 4 consecutive days.

Flow cytometric analysis and sorting

Single cell suspension was prepared from lung or other tissues. About $2 \times 10^{6}$ cells were incubated with anti-CD16/CD32 before staining with fluorochrome-conjugated antibodies. Dead cells were excluded using cell viability dye (The live/dead fixable far-red dead cell stain kit, Invitrogen). For staining of transcriptional factor, cells were fixed and permeabilized (Foxp3/Transcription Factor Staining Buffer Set, eBioscience), stained with surface markers before incubation with fluorochrome-conjugated antibody against transcriptional factor. For analysis of PPARy expression, cells were fixed and permeabilized followed by staining with rabbit antiPPAR $y$ western blot antibody, then labeled with fluorochromeconjugated goat anti-rabbit IgG secondary antibody. For measuring intracellular cytokine expression, cells were stimulated with 50 $\mathrm{ng} / \mathrm{ml}$ PMA (Sigma-Aldrich), $1 \mu \mathrm{g} / \mathrm{ml}$ ionomycin (Sigma-Aldrich), and $1 \mu \mathrm{g} / \mathrm{ml}$ brefeldin A in complete RPMl-1640 for $4 \mathrm{~h}$. Cells were labeled with antibodies to surface markers, fixed and permeabilized using an intracellular fixation and permeability kit (eBioscience), then stained with antibodies against cytokines. LSRFortessa flow cytometer (BD Bioscience) was used for samples acquiring, and data were analyzed with Flowjo V10.4. To sort mouse ILC2, cells were depleted of T, B, NK, myeloid, and erythroid lineages by labeling with biotin-conjugated anti-CD3e, anti-CD4,

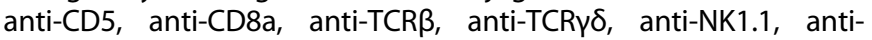
CD45R/B220, anti-CD11b, anti-Ly6G, anti-Erythroid marker (TER119), anti-CD11c, followed by streptavidin-paramagnetic particles
(BD Bioscience) according to manufacturer's instructions. The harvested cells were labeled with fluorochrome-conjugated antibodies $\left(\mathrm{CD} 45^{+} \mathrm{Lin}^{-} \mathrm{CD} 90.2^{+} \mathrm{CD} 25^{+}\right)$and sorted by Aria III (BD Bioscience). For human ILC2 sorting, PBMCs were labeled with biotin-conjugated anti-CD2, anti-CD3, anti-CD10, anti-CD11b, antiCD14, anti-CD16, anti-CD19, anti-CD56, anti-CD123, and antiCD235a to deplete T cells, B cells, NK cells, myeloid cells, granulocytes, and red blood cells, followed by streptavidinparamagnetic particles. Then, the collected lineage-negative cells were stained with the specific fluorochrome-conjugated antibodies for ILC2s $\left(\mathrm{CD} 45^{+} \mathrm{Lin}^{-} \mathrm{CD} 127^{+} \mathrm{CRTH} 2^{+} \mathrm{CD} 161^{+}\right)$. The purity of cell sorting was $\geq 95 \%$. Antibodies used are listed in Table S1.

Adoptive transfer of ILC2

Lung ILC2s were sorted from IL-33 i.p. treated PPAR $\gamma^{\mathrm{fl} / \mathrm{fl}}$ and PPAR ${ }^{\mathrm{fl} / \mathrm{fl}}$ Vav $1^{\text {Cre }}$ mice, and rested in complete medium for $24 \mathrm{~h}$, before intravenously transferred into NCG mice, respectively $\left(1.5 \times 10^{4}\right.$ cells/mouse). Mice were then challenged with IL-33 intranasally for 3 consecutive days. ${ }^{7}$ Mice were sacrificed and analyzed $24 \mathrm{~h}$ later after the last challenge.

\section{Cell isolation from tissues}

To isolate cells from BALF and lung tissue, lungs were flushed by $0.5 \mathrm{ml}$ cold PBS twice via a thin tube inserted into a cut made in the trachea as described. Then, lungs were perfused with $20 \mathrm{ml}$ cold PBS. Lung tissues were cut into small pieces and digested with $0.5 \mathrm{mg} / \mathrm{ml}$ type I collagenase (Invitrogen, USA) in RPMI-1640 for $1 \mathrm{~h}$ at $37^{\circ} \mathrm{C}$ with continuous agitation in an incubator. The crude suspensions were further filtered through $70 \mu \mathrm{m}$ cell strainers. Mononuclear cells were obtained by a $40 \% / 80 \%$ Percoll (GE Healthcare, Uppsala, Sweden) gradient. Splenocytes and $\mathrm{mLN}$ cells were obtained by mechanical disruption on $70 \mu \mathrm{m}$ cell strainers. Isolation of cells from large intestine was performed by digestion with $0.5 \mathrm{mg} / \mathrm{ml}$ collagenase I and $5 \mathrm{U} / \mathrm{ml}$ DNase for 40 min as described.

\section{Human PBMC isolation}

This study was approved by the Ethics Review Board of Sun Yat-sen University. Human peripheral blood mononuclear cells (PBMCs) from fresh blood samples were isolated by Ficoll centrifugation as described. ${ }^{59}$ Isolated PBMCs were washed and resuspended in cold PBS containing $1 \%$ FBS before subsequent use.

\section{ILC2 culture in vitro}

For ILC2 function analysis in vitro, $5 \times 10^{3}$ lung ILC2 were cultured in the presence of $20 \mathrm{ng} / \mathrm{ml} \mathrm{IL}-33,10 \mathrm{ng} / \mathrm{ml} \mathrm{IL-2,} \mathrm{and} 20 \mathrm{ng} / \mathrm{ml} \mathrm{IL-7}$ for 3 days, $^{7}$ the supernatants were collected to measure IL-5 and IL-13 by ELISA. For rosiglitazone and GW9662 treatments, both were used at concentration of $10 \mu \mathrm{M} .^{60,61}$

\section{Quantitative real-time PCR (qRT-PCR)}

Total mRNA were extracted using TRIzol (Invitrogen) and reverse transcribed with a synthesis kit (Takara). Genes mRNA expression were analyzed by qPCR. The primer sequences were listed in Table S2.

\section{C. rodentium model}

Mice were orally gavaged with $2 \times 10^{9} \mathrm{CFUs}$ of $C$. rodentium ${ }^{44}$ and were monitored daily to record weight loss. Mice were sacrificed on day 6 , the colon length were measured. The cytokine production of ILC1 and ILC3 from colon lamina propria was evaluated by flow cytometry.

Retroviral transduction

Retroviral transduction was performed as described previously. Lung ILC2 were sorted from PPAR $\gamma^{\mathrm{fl} / \mathrm{fl}}$ and PPAR $\gamma^{\mathrm{fl} / \mathrm{fl}}$ Vav $1^{\mathrm{Cre}}$ mice and treated with retrovirus-containing supernatants supplemented with polybrene $(8 \mu \mathrm{g} / \mathrm{ml})$ in 96 -well plate, centrifuged at 
$1000 \times g$ for $2 \mathrm{~h}$ at $32^{\circ} \mathrm{C}$, then cultured at $37^{\circ} \mathrm{C}$ for another $6 \mathrm{~h}$. Cells were washed and cultured in fresh complete medium with $10 \mathrm{ng} / \mathrm{ml}$ of IL-2, IL-7, and IL-33 for 3 days. The efficiency of retroviral infection was evaluated by GFP $(+)$ cells using flow cytometry. The supernatants were collected for the analysis of IL-5 and IL-13 by ELISA.

\section{Chromatin immunoprecipitation (ChIP)}

Lineage negative cells were sorted from lung of WT mice, ChIP assay was conducted as previously described. Briefly, Lineage negative cells were fixed with a $1 \%$ formaldehyde solution, lysed, and sheared by sonication. Cell lysates were precleared with protein G-agarose and immunoprecipitated with anti-PPARy antibodies or an anti-lgG control. Antibody-chromatin complexes were collected with protein G-agarose. The DNA was purified and quantified by PCR using indicated primers (Table S2). $10 \%$ of the lysate before immunoprecipitation was used as the input control. Data are presented as the folds of immunoprecipitated to input cycle threshold values.

\section{Statistics}

All data are derived from at least two independent experiments. Statistical analysis was performed with GraphPad Prism 8.0. Results show mean \pm SEM and statistical significance was determined by a two-tailed unpaired Student's $t$ test or one-way ANOVA with Bonferroni post-test. $P$ value $<0.05$ was considered significant.

\section{ACKNOWLEDGEMENTS}

This work was supported by the following grants to J.Z.: National Natural Science Foundation of China Grants 81925018, 81771665 (to J.Z.); the Start-Up Fund for HighLevel Talents of Tianjin Medical University (to J.Z.).

\section{AUTHOR CONTRIBUTIONS}

Q.X., J.H., and A.L. performed the experiments and analyzed the data. H.X., L.Z., and P. Z. participated in experiments. G.J. provided suggestions for experimental design. J.Z. conceptualized, supervised, interpreted the experiments, and wrote the paper.

\section{ADDITIONAL INFORMATION}

The online version of this article (https://doi.org/10.1038/s41385-020-00339-6) contains supplementary material, which is available to authorized users.

Competing interests: The authors declare no competing interests.

Publisher's note Springer Nature remains neutral with regard to jurisdictional claims in published maps and institutional affiliations.

\section{REFERENCES}

1. Papi, A., Brightling, C., Pedersen, S. E. \& Reddel, H. K. Asthma. Lancet 391, 783-800 (2018).

2. Lambrecht, B. N. \& Hammad, H. The immunology of asthma. Nat. Immunol. 16, 45-56 (2015)

3. Divekar, R. \& Kita, H. Recent advances in epithelium-derived cytokines (IL-33, IL25 , and thymic stromal lymphopoietin) and allergic inflammation. Curr. Opin. Allergy Clin. Immunol. 15, 98-103 (2015).

4. Monticelli, L. A., Sonnenberg, G. F. \& Artis, D. Innate lymphoid cells: critical regulators of allergic inflammation and tissue repair in the lung. Curr. Opin. Immunol. 24, 284-289 (2012).

5. Halim, T. Y. et al. Group 2 innate lymphoid cells are critical for the initiation of adaptive $\mathrm{T}$ helper 2 cell-mediated allergic lung inflammation. Immunity 40, 425-435 (2014)

6. Mirchandani, A. S. et al. Type 2 innate lymphoid cells drive CD4+ Th2 cell responses. J. Immunol. 192, 2442-2448 (2014).

7. Lei, A. H. et al. ICAM-1 controls development and function of ILC2. J. Exp. Med. 215, 2157-2174 (2018)

8. Serafini, N., Vosshenrich, C. A. \& Di Santo, J. P. Transcriptional regulation of innate lymphoid cell fate. Nat. Rev. Immunol. 15, 415-428 (2015).
9. Gasteiger, G., Fan, X., Dikiy, S., Lee, S. Y. \& Rudensky, A. Y. Tissue residency of innate lymphoid cells in lymphoid and nonlymphoid organs. Science 350 981-985 (2015).

10. Walker, J. A., Barlow, J. L. \& McKenzie, A. N. J. Innate lymphoid cells-how did we miss them? Nat. Rev. Immunol. 13, 75-87 (2013).

11. Kabata, H., Moro, K. \& Koyasu, S. The group 2 innate lymphoid cell (ILC2) regulatory network and its underlying mechanisms. Immunol. Rev. 286, 37-52 (2018).

12. Brestoff, J. R. et al. Group 2 innate lymphoid cells promote beiging of white adipose tissue and limit obesity. Nature 519, 242-246 (2015).

13. Monticelli, L. A. et al. Innate lymphoid cells promote lung-tissue homeostasis after infection with influenza virus. Nat. Immunol. 12, 1045-1054 (2011).

14. Vivier, E. et al. Innate lymphoid cells: 10 years on. Cell 174, 1054-1066 (2018).

15. Hurrell, B. P., Shafiei Jahani, P. \& Akbari, O. Social networking of group two innate lymphoid cells in allergy and asthma. Front. Immunol. 9, 2694 (2018).

16. Hoyler, T. et al. The transcription factor GATA-3 controls cell fate and maintenance of type 2 innate lymphoid cells. Immunity 37, 634-648 (2012).

17. Mjosberg, J. et al. The transcription factor GATA3 is essential for the function of human type 2 innate lymphoid cells. Immunity 37, 649-659 (2012).

18. Clark, R. B. The role of PPARs in inflammation and immunity. J. Leukoc. Biol. 71 388-400 (2002).

19. Ahmadian, M. et al. PPARgamma signaling and metabolism: the good, the bad and the future. Nat. Med. 19, 557-566 (2013).

20. Tontonoz, P., Hu, E. \& Spiegelman, B. M. Stimulation of adipogenesis in fibroblasts by PPAR gamma 2, a lipid-activated transcription factor. Cell 79, 1147-1156 (1994).

21. Daynes, R. A. \& Jones, D. C. Emerging roles of PPARs in inflammation and immunity. Nat. Rev. Immunol. 2, 748-759 (2002).

22. Honda, K., Marquillies, P., Capron, M. \& Dombrowicz, D. Peroxisome proliferatoractivated receptor gamma is expressed in airways and inhibits features of airway remodeling in a mouse asthma model. J. Allergy Clin. Immunol. 113, 882-888 (2004).

23. Benayoun, L. et al. Regulation of peroxisome proliferator-activated receptor gamma expression in human asthmatic airways: relationship with proliferation, apoptosis, and airway remodeling. Am. J. Respir. Crit. Care Med. 164, 1487-1494 (2001).

24. Li, W. et al. Association of peroxisome proliferator-activated receptor-gamma gene polymorphisms and gene-gene interaction with asthma risk in a Chinese adults population. Int. J. Clin. Exp. Med. 8, 19346-19352 (2015).

25. Zhang, Y., Wang, Z. \& Ma, T. Associations of genetic polymorphisms relevant to metabolic pathway of vitamin D3 with development and prognosis of childhood bronchial asthma. DNA Cell Biol. 36, 682-692 (2017).

26. Spears, M. et al. Bronchodilatory effect of the PPAR-gamma agonist rosiglitazone in smokers with asthma. Clin. Pharm. Ther. 86, 49-53 (2009).

27. Banno, A., Reddy, A. T., Lakshmi, S. P. \& Reddy, R. C. PPARs: key regulators of airway inflammation and potential therapeutic targets in asthma. Nucl. Receptor Res. 5, 101306 (2018)

28. Narala, V. R. et al. Pioglitazone is as effective as dexamethasone in a cockroach allergen-induced murine model of asthma. Respir. Res. 8, 90 (2007).

29. Lee, S. Y. et al. Peroxisome proliferator-activated receptor-gamma inhibits cigarette smoke solution-induced mucin production in human airway epithelial (NCl-H292) cells. Am. J. Physiol. Lung Cell. Mol. Physiol. 291, L84-L90 (2006).

30. Schneider, C. et al. Alveolar macrophages are essential for protection from respiratory failure and associated morbidity following influenza virus infection. PLoS Pathog. 10, e1004053 (2014).

31. Hammad, $\mathrm{H}$. et al. Activation of peroxisome proliferator-activated receptor gamma in dendritic cells inhibits the development of eosinophilic airway inflammation in a mouse model of asthma. Am. J. Pathol. 164, 263-271 (2004).

32. Nobs, S. P. et al. PPARgamma in dendritic cells and T cells drives pathogenic type2 effector responses in lung inflammation. J. Exp. Med. 214, 3015-3035 (2017).

33. Chen, T. et al. PPAR- $\gamma$ promotes type 2 immune responses in allergy and nematode infection. Sci. Immunol. 2, pii: eaal5196 (2017).

34. Pokrovskii, M. et al. Characterization of transcriptional regulatory networks that promote and restrict identities and functions of intestinal innate lymphoid cells. Immunity 51, 185-197. e186 (2019).

35. Karagiannis, F. et al. Lipid-droplet formation drives pathogenic group 2 innate lymphoid cells in airway inflammation. Immunity 52, 620-634. e626 (2020).

36. Taylor, S. et al. PD-1 regulates KLRG1(+) group 2 innate lymphoid cells. J. Exp. Med. 214, 1663-1678 (2017).

37. Batyrova, B. et al. PD-1 expression affects cytokine production by ILC2 and is influenced by peroxisome proliferator-activated receptor-gamma. Immun. Inflamm. Dis. 8, 8-23 (2020).

38. Robinette, M. L. et al. Transcriptional programs define molecular characteristics of innate lymphoid cell classes and subsets. Nat. Immunol. 16, 306-317 (2015).

39. Ricote, M. et al. Expression of the peroxisome proliferator-activated receptor gamma (PPARgamma) in human atherosclerosis and regulation in macrophages 
by colony stimulating factors and oxidized low density lipoprotein. Proc. Natl Acad. Sci. USA 95, 7614-7619 (1998).

40. Salimi, M. et al. A role for IL-25 and IL-33-driven type-2 innate lymphoid cells in atopic dermatitis. J. Exp. Med. 210, 2939-2950 (2013).

41. Hazenberg, M. D. \& Spits, H. Human innate lymphoid cells. Blood 124, 700-709 (2014).

42. Huang, Y. et al. IL-25-responsive, lineage-negative KLRG1(hi) cells are multipotential 'inflammatory' type 2 innate lymphoid cells. Nat. Immunol. 16, 161-169 (2015).

43. Abt, M. C. et al. Innate immune defenses mediated by two ILC subsets are critical for protection against acute clostridium difficile infection. Cell Host Microbe 18, 27-37 (2015).

44. Satoh-Takayama, N. et al. The chemokine receptor CXCR6 controls the functional topography of interleukin-22 producing intestinal innate lymphoid cells. Immunity 41, 776-788 (2014).

45. Ebbo, M., Crinier, A., Vely, F. \& Vivier, E. Innate lymphoid cells: major players in inflammatory diseases. Nat. Rev. Immunol. 17, 665-678 (2017).

46. Li, B. W. et al. T cells are necessary for ILC2 activation in house dust mite-induced allergic airway inflammation in mice. Eur. J. Immunol. 46, 1392-1403 (2016).

47. Liu, B., Lee, J. B., Chen, C. Y., Hershey, G. K. \& Wang, Y. H. Collaborative interactions between type 2 innate lymphoid cells and antigen-specific CD4+ Th2 cells exacerbate murine allergic airway diseases with prominent eosinophilia. J. Immunol. 194, 3583-3593 (2015).

48. Moriyama, $\mathrm{S}$. et al. beta2-adrenergic receptor-mediated negative regulation of group 2 innate lymphoid cell responses. Science 359, 1056-1061 (2018).

49. Guan, H. P., Ishizuka, T., Chui, P. C., Lehrke, M. \& Lazar, M. A. Corepressors selectively control the transcriptional activity of PPARgamma in adipocytes. Genes Dev. 19, 453-461 (2005).

50. Matsusue, K. et al. Hepatic steatosis in leptin-deficient mice is promoted by the PPARgamma target gene Fsp27. Cell Metab. 7, 302-311 (2008).
51. Anderson, J. R. et al. Evaluation of the PPAR-gamma agonist pioglitazone in mild asthma: a double-blind randomized controlled trial. PLOS ONE 11, e0160257 (2016).

52. Kaler, M. et al. A randomized, placebo-controlled, double-blinded, crossover trial of pioglitazone for severe asthma. J. Allergy Clin. Immunol. 140, 1716-1718 (2017).

53. Richards, D. B., Bareille, P., Lindo, E. L., Quinn, D. \& Farrow, S. N. Treatment with a peroxisomal proliferator activated receptor gamma agonist has a modest effect in the allergen challenge model in asthma: a randomised controlled trial. Respir. Med. 104, 668-674 (2010).

54. Wilhelm, C. et al. Critical role of fatty acid metabolism in ILC2-mediated barrier protection during malnutrition and helminth infection. J. Exp. Med. 213, 1409-1418 (2016).

55. Poulsen, L., Siersbaek, M. \& Mandrup, S. PPARs: fatty acid sensors controlling metabolism. Semin. Cell Dev. Biol. 23, 631-639 (2012).

56. Lee, M. W. et al. Activated type 2 innate lymphoid cells regulate beige fat biogenesis. Cell 160, 74-87 (2015).

57. Maazi, H. et al. ICOS:ICOS-ligand interaction is required for type 2 innate lymphoid cell function, homeostasis, and induction of airway hyperreactivity. Immunity 42, 538-551 (2015).

58. Monticelli, L. A. et al. Arginase 1 is an innate lymphoid-cell-intrinsic metabolic checkpoint controlling type 2 inflammation. Nat. Immunol. 17, 656-665 (2016).

59. Qin, A. et al. Expansion of monocytic myeloid-derived suppressor cells dampens $T$ cell function in HIV-1-seropositive individuals. J. Virol. 87, 1477-1490 (2013).

60. Wan, J. et al. Pioglitazone modulates the proliferation and apoptosis of vascular smooth muscle cells via peroxisome proliferators-activated receptor-gamma. Diabetol. Metab. Syndr. 6, 101 (2014).

61. Whiteside, C. et al. Rosiglitazone prevents high glucose-induced vascular endothelial growth factor and collagen IV expression in cultured mesangial cells. Exp. Diabetes Res. 2009, 910783 (2009). 